\title{
Acid-Base Equilibrium Constant and Dipole Moment of Tribenzylammonium Picrate in Benzene from Meas- urements of Dielectric Constant
}

\author{
By Arthur A. Maryott
}

\begin{abstract}
The equilibrium constant for the dissociation of tribenzylammonium picrate into picric acid and tribenzylamine in benzene at $30^{\circ}$ and $40^{\circ} \mathrm{C}$ has been determined from the variation of dielectric constant with concentration. The heat of reaction is in the neighborhood of 11.4 kilocalories per mole. The dipole moment of tribenzylammonium picrate, $12.0 \times 10^{-18} \mathrm{esu}$, is substantially the same as found previously for the picrates of the more strongly basic alkylamines. The dipole moment of picric acid, $1.75 \times 10^{-18} \mathrm{esu}$, was also determined.
\end{abstract}

\section{Introduction}

The measurement of dielectric constant has been used rather infrequently as a quantitative method for studying association and other mass law equilibria in dilute solutions. The procedure, however, has been employed successfully to determine the dimerization constants of a number of carboxylic acids in benzene [1]. ${ }^{1}$ Although the method has more limited application than do methods based on colligative properties, it has certain advantages and, in particular, is free from assumptions regarding the validity of Raoult's law.

In this investigation, the measurement of dielectric constant is used to determine the equilibrium constant for the reaction between picric acid and a relatively weak tertiary amine, tribenzylamine, in benzene. Such acid-base equilibria are well suited for study because of the relatively large dipole moment of the salt compared to that of the component acid and base. In a previous investigation of the picrates of various alkylamines of sufficient basic strength to prevent measurable dissociation into free acid and base [2], it was found that the picrates of tertiary amines, but not those of primary and secondary amines, showed no significant association in benzene. Consequently, association of the salt should not be a complicating factor in the reaction with tribenzylamine.

${ }_{1}^{1}$ Figures in brackets indicate the literature references at the end of this paper.

\section{Equipment and Procedure}

The equipment and procedure have been described [2]. Tribenzylammonium picrate was prepared in the same manner as the other picrates. The melting point was $190^{\circ} \mathrm{C}$. Because of the rather low solubility of the picrate in benzene, only the more dilute solutions were prepared in the usual manner with the addition of stock solution directly to the solvent or solution in the cell. The more concentrated solutions were prepared in flasks and introduced separately into the cell.

\section{Experimental Results}

The experimental data obtained at $30^{\circ}$ and at $40^{\circ} \mathrm{C}$ are given in the first three columns of table $1 . \quad N_{2}$ is the stoichiometric mole fraction of the solute and $\Delta \epsilon$ the difference in dielectric constant between the solution and the solvent.

The gradual decrease in the quantity, $\Delta \epsilon / N_{2}$, with increasing dilution indicates that the salt is partly dissociated into picric acid and tribenzylamine. The equilibrium constant for this reaction, $B z_{3} N H \ldots \bar{P} i=B z_{3} N+H P i$, can be determined by a procedure similar to that described previously [2]. Regarding the change in dielectric constant as the sum of three independent contributions that are linear functions of the concentrations of the acid, base, and salt, respectively,

$$
\Delta \epsilon=k_{A} \alpha N_{2}+k_{B} \alpha N_{2}+k_{S}(1-\alpha) N_{2}
$$

or

$$
\alpha=\frac{k_{S}-\Delta \epsilon / N_{2}}{k_{S}-k_{A}-k_{B}}
$$


TABle 1. Experimental data and acid-base equilibrium constant for tribenzylammonium picrate in benzene

\begin{tabular}{|c|c|c|c|c|}
\hline$N_{2} \times 10^{4}$ & $\Delta \epsilon$ & $\Delta \epsilon / N_{2}$ & $\alpha$ & $K_{N} \times 10^{3}$ \\
\hline \multicolumn{5}{|c|}{$30^{\circ} \mathrm{C}$} \\
\hline 0.899 & 0.00739 & 82.2 & 0.591 & 7.68 \\
\hline 1. 081 & $.0093_{8}$ & 86.8 & .566 & 7.98 \\
\hline 1. 585 & $.0159_{7}$ & 100.7 & .491 & 7. 51 \\
\hline 1. 698 & $.0173_{7}$ & 102.3 & .483 & 7.66 \\
\hline 2. 327 & \multirow{2}{*}{$.0262_{1}$} & 112.6 & .427 & 7.41 \\
\hline 3. 467 & & 121.1 & .381 & 8.13 \\
\hline 4. 127 & .0525 & 127.2 & .349 & 7.72 \\
\hline 7. 038 & .0985 & 140.0 & .280 & 7.66 \\
\hline \multicolumn{3}{|c|}{$k_{A}+k_{B}=6.2$} & \multicolumn{2}{|c|}{$K_{N}=7.71 \times 10^{-5 \mathrm{a}}$} \\
\hline \multicolumn{5}{|c|}{$40^{\circ} \mathrm{C}$} \\
\hline 0.907 & $0.0054_{b}$ & 60.2 & 0.699 & 14. 7 \\
\hline 1. 613 & $.0127_{7}$ & 79.2 & .594 & 14.0 \\
\hline 2. 186 & $.0194_{8}$ & 89.1 & .539 & 13.8 \\
\hline 4. 126 & .0441 & 106. 9 & 440 & 14.3 \\
\hline 4. 986 & .0554 & 111.1 & .417 & 14.9 \\
\hline 5. 867 & .0695 & 118.5 & .376 & 13. 3 \\
\hline 7. 500 & .0914 & 121.9 & .357 & 14.9 \\
\hline 8. 198 & .1019 & 124.3 & .343 & 14.7 \\
\hline \multicolumn{2}{|c|}{$k_{A}+k_{B}=6.0$} & $=186.2^{\mathrm{a}}$ & $K_{N}=14$. & $10^{-5 \text { a }}$ \\
\hline
\end{tabular}

a Obtained from least squares solution of eq 3 , as shown in figure 1 .

where $\alpha$ is the fraction of salt dissociated, and $k_{A}, k_{B}$, and $k_{S}$ are the proportionality constants for the acid, base, and salt, respectively. $k_{A}$ and $k_{B}$ were determined experimentally at $30^{\circ} \mathrm{C}$ from the dielectric constants of dilute solutions of picric acid and of tribenzylamine and then calculated for $40^{\circ} \mathrm{C}$ on the assumption that the dipole moment remains unchanged. The expression for the equilibrium constant,

$$
K_{N}=\frac{\left(k_{S}-\Delta \epsilon / N_{2}\right)^{2} N_{2}}{\left(\Delta \epsilon / N_{2}-k_{A}-k_{B}\right)\left(k_{S}-k_{A}-k_{B}\right)},
$$

then contains only one unknown quantity in addition to $K_{N}$. In order to solve for $k_{S}$ and $K_{N}$, it is convenient to rearrange eq 2 in the following linear form,

$$
\Delta \epsilon / N_{2}+\left[K_{N}\left(k_{S}-k_{A}-k_{B}\right)\right]^{1 / 2} Z-k_{S}=0,
$$

where

$$
Z=\left[\left(\Delta \epsilon / N_{2}-k_{A}-k_{B}\right) / N_{2}\right]^{1 / 2} .
$$

The solution obtained by the method of least squares is shown graphically in figure 1, where $\Delta \epsilon / N_{2}$ is plotted as a function of 7 . The intercept yields $k_{S}$. $K_{N}$ is then determined from the

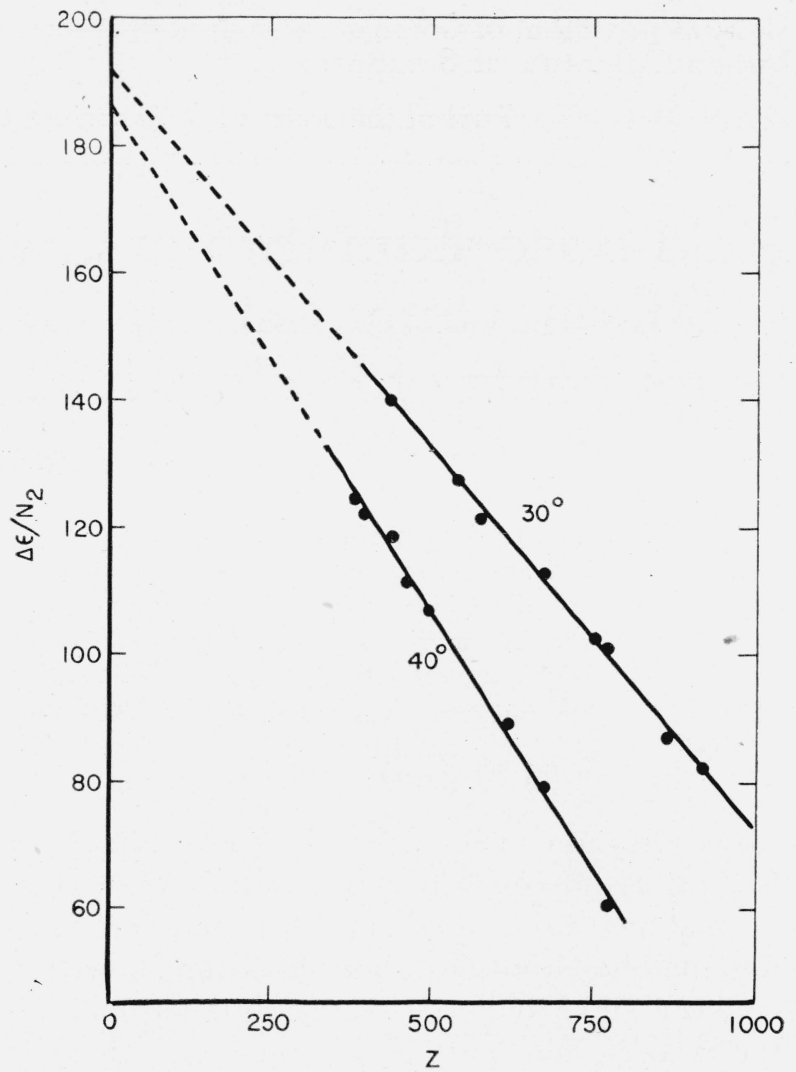

FiguRE 1. Graphical representation of data for tribenzylammonium picrate in benzene in accordance with equations 3 and 4.

slope of the line. These values are included in table 1. For comparison, values of $\alpha$ and of $K_{N}$ at each concentration ${ }^{2}$ are given, respectively, in the fourth and fifth columns.

There appear to be few or no data in the literature on the heats of reaction, or neutralization, of picric acid with amines in inert media, although such data should prove of interest with regard to relative basic strengths. The heat of reaction for picric acid and tribenzylamine, calculated from the equilibrium constant at the two temperatures, is in the neighborhood of $11.4 \mathrm{kcal} / \mathrm{mole}$.

The dipole moment of tribenzylammonium picrate, calculated from $k_{S}$ at either $30^{\circ}$ or $40^{\circ} \mathrm{C}$, is $12.0 \times 10^{-18} \mathrm{esu}$. As the dipole moment of picric acid apparently has not been reported previously, the experimental data are given in table 2 . The various symbols have been defined either in this or the previous paper. The moment of picric acid (1.75)

2 The equilibrium constants also may be expressed in moles per liter rather than in mole fraction by multiplying $K_{N}$ by the factor, $(1,000 d) / M$, where, as a sufficient approximation for the dilute solutions involved, $d$ is the density of the solvent and $M$ its molecular weight. 
appears to be a little larger than that of phenol (1.5 to 1.6) [3]. The value calculated for picric acid, however, may be subject to greater uncertainty, because of the neglect of atomic polarization, than is usually the case. Nonpolar molecules containing highly polar but symmetrically. placed, substituents frequently have a relatively high atomic polarization [4]. If the atomic polarization is assumed to be the same as that of $2,4,6$ trinitrobenzene [5], the moment calculated for picric acid is 1.57 .

TABle 2. Experimental data $\left(30^{\circ} \mathrm{C}\right)$ and dipole moment, $\mu$, of picric acid in benzene

\begin{tabular}{|c|c|c|}
\hline$N_{2} \times 10^{3}$ & $\Delta \epsilon$ & $\Delta \epsilon / N_{2}\left(k_{A}\right)$ \\
\hline & & 4.58 \\
0.000 & & 4.58 \\
1.769 & 0.0081 & 4.58 \\
5.522 & .0253 & 4.57 \\
9.152 & .0418 & 4.60 \\
12.40 & .0570 & \\
& & \\
& \multicolumn{3}{|c|}{$\Delta d / N_{2}=1.31$} \\
$P_{2}=106.0$ & $P_{B}=44.6 \quad \mu=1.75 \times 10^{-18} \mathrm{esu}$. \\
\hline \multicolumn{3}{c}{} \\
\hline
\end{tabular}

\section{Discussion}

It has been suggested [6] that tribenzylammonium picrate may exist in tautomeric equilib- rium between two forms in media of low dielectric constant, one form being a true salt and the other a molecular addition compound. In view of the fact that the moment of tribenzylammonium picrate is practically the same as for the trialkylammonium picrates, this possibility seems unlikely. Acids, however, do form hydrogen bonded complexes rather than salts with certain very weak bases. An interesting question arises as to whether, as the strength of the base is decreased, the transition from salt formation to hydrogen bonding occurs gradually or abruptly. Although tribenzylamine is a substantially weaker base than the trialkylamines, the proton transfer from acid to base appears to be equally complete in the two cases.

\section{References}

[1] H. A. Pohl, M. E. Hobbs, and P. M. Gross, J. Chem. Phys. 9, 408 (1941); A. A. Maryott, M. E. Hobbs, and P. M. Gross, J. Chem. Phys. 9, 415 (1941).

[2] A. A. Niaryott, J. Research NBS 41, 1 (1948) RP1896.

[3] H. L. Donle and K. A. Gehrckens, Z. physik. Chem. [B] 18, 316 (1932); A. R. Martin, Nature 135, 909 (1935).

[4] I. E. Coop and L. E. Sutton, J. Chem. Soc. 1938, 1269.

[5] R. J. W. LeFevre and C. G. LeFevre, J. Chem. Soc. $1935,957$.

[6] M. A. Elliott and R. M. Fuoss, J. Am. Chem. Soc. 61, 294 (1939).

Washington, April 16, 1948. 